\title{
Strategies for the rapid prenatal diagnosis of chromosome aneuploidy
}

\author{
Kathy Mann ${ }^{*, 1}$, Celia Donaghue ${ }^{1}$, Susan P Fox ${ }^{1}$, Zoe Docherty ${ }^{1}$ and \\ Caroline Mackie Ogilvie ${ }^{1}$
}

\author{
${ }^{1}$ Cytogenetics Department, Guy's \& St Thomas' Hospital Trust, London, UK
}

Rapid diagnosis of common chromosome aneuploidies in raised risk pregnancies, usually prior to full karyotype analysis, is now carried out in a number of European genetic centres; several techniques for detecting genomic copy number changes have been described. Prenatal diagnosis of genetic disease requires accurate and robust assays; the invasive procedures are associated with a risk of pregnancy loss and an abnormal result may lead to termination of the pregnancy. The testing of prenatal material (amniotic fluid, chorionic villi or, more rarely, fetal blood) is associated with specific problems, including the quality and quantity of the tissue and difficulties of interpretation due to phenomena such as maternal cell contamination and mosaicism. In addition, there are 24-h, high-throughput demands on centres offering such a service. The extent to which existing and proposed strategies, including different PCRbased assays, a multiplex ligation-dependent probe amplification approach, and microarrays, fulfil the requirements of rapid prenatal testing is discussed. In the past 3 years, we have tested 7720 prenatal samples for trisomies 13, 18 and 21 using a quantitative fluorescence-PCR (QF-PCR) approach. The abnormality rate was $5.7 \%$. There were no misdiagnoses for nonmosaic trisomy, the amplification failure rate was $0.09 \%$ of samples, and $97 \%$ of samples received a report on the working day following sample receipt. Maternal cell contamination and mosaicism were also detected. Our data recommend a QF-PCR approach as the current method of choice for rapid aneuploidy testing.

European Journal of Human Genetics (2004) 12, 907-915. doi:10.1038/sj.ejhg.5201224

Published online 4 August 2004

Keywords: QF-PCR; prenatal diagnosis; Down syndrome; aneuploidy; MLPA; microarrays

\section{Introduction}

Prenatal diagnosis of pregnancies at raised risk of a chromosome abnormality is carried out by karyotype analysis of cultured cells, with average reporting times in the UK of 13-14 days. ${ }^{1}$ The majority of chromosome abnormalities identified in prenatal samples are trisomy for chromosomes 13, 18 or 21 and sex chromosome aneuploidies. These are associated with the newborn phenotypes, Patau syndrome, Edwards syndrome and Down syndrome (trisomy 13, 18 and 21 respectively), and the

*Correspondence: Dr K Mann, Cytogenetics Department, 5th Floor, Guy's Tower, St Thomas Street, London SE1 9RT, UK. Tel: + 44-20-7188-1710; Fax: + 44-20-7188-1697; E-mail: Kathy.Mann@gstt.sthames.nhs.uk Received 15 January 2004; revised 11 March 2004; accepted 16 April 2004 less severe Turner (monosomy X) and Klinefelter (XXY) syndromes.

To reduce maternal anxiety and improve pregnancy management, more rapid aneuploidy testing is now the aim of many European genetic testing centres, and the UK National Screening Committee has recommended that a rapid result should be offered as part of the UK National Health Service provision to all women undergoing invasive testing. Although genomic copy number can be measured in a number of ways, a rapid prenatal aneuploidy test should fulfil certain criteria. First and foremost, the assay must be accurate, with a minimum number of falsenegative results, and should have safeguards in place to ensure there are no false positives, as pregnancies may be terminated following a rapid abnormal result. The assay 
must be robust with few ambiguous results and failed tests, as these may increase parental anxiety. To be of value, the service should be rapid and capable of high sample throughput. Ideally, capital, consumable and labour costs should be low, as the rapid test is usually carried out in addition to full karyotype analysis.

Additionally, any diagnostic strategy must take into account complications associated with the testing of prenatal material (amniotic fluid, chorionic villi and fetal blood). Sample quantity is limited for all sample types, but especially amniocentesis procedures carried out at an early gestation and clinically difficult CVS procedures. Sample quality is variable, particularly in amniotic fluid samples where up to $40 \%$ have some blood-staining (unpublished data). This blood-staining is sometimes visible in the fluid, but is otherwise evident upon examination of the cell pellet and if due to an old bleed is brown/green in colour, often an indication of very poor sample quality.

Maternal cell contamination (MCC) of fetal material may arise during any of the invasive sampling procedures. Although the presence of maternal material in CVS can be reduced and usually eliminated by thorough removal of maternal decidua, maternal cells (usually lymphocytes, more rarely fibroblasts from a maternal tissue plug) cannot be easily removed from amniotic fluid and fetal blood. It is therefore important to identify samples with MCC in order to avoid a diagnosis based on maternal cells. Mosaicism for a chromosome abnormality is also a widely reported feature of prenatal samples, with mosaicism for trisomy 13,18 or 21 occurring in approximately $0.26 \%$ of CVS. ${ }^{2}$ Ideally, minority normal and abnormal cell lines should be identified as these may be of clinical significance to the pregnancy.

Several techniques have been applied to the rapid diagnosis of aneuploidy in prenatal samples. Karyotype analysis of spontaneous metaphases in the cytotrophoblast layer of uncultured CVS (direct preparations) permits a whole genome scan to be carried out, albeit at a low resolution, which may additionally detect structural chromosome abnormalities. However, only CVS can be analysed, failure rates are significant, protocols are timeconsuming and confined placental mosaicism complicates interpretation; ${ }^{2}$ alternative approaches have therefore been developed. The most widely established method is interphase-fluorescence in situ hybridisation (FISH). ${ }^{3,4}$ Here, a set of chromosome-specific fluorescence-labelled probes are hybridised to interphase nuclei of uncultured prenatal cells; the number of fluorescent signals in each nucleus represents chromosome copy number. Between 50 and 100 cells are usually analysed to allow for low-level background and signal overlay that can occur during FISH procedures. Commercial probe sets, approved by the Federal Drug Administration (FDA), are available. Many UK cytogenetic laboratories use this interphase-FISH approach but restrict its application to a subset of prenatal referrals due to its significant cost and labour requirements.

A QF-PCR approach ${ }^{5,6}$ is a more recent addition to aneuploidy diagnosis. The technique involves the relative quantification of microsatellite alleles to determine sequence copy number; amplification using fluorescencelabelled primers is followed by size separation and allele peak measurement on a semi-automated genetic analyser. Several assays have been developed and applied to prenatal diagnosis. ${ }^{7-11}$ The number and variety of microsatellite markers multiplexed together differs between assays and determines assay efficiency. As with most PCR-based assays, this approach is capable of generating fast results using economical in-house assays and minimum labour requirements. However, the development of reliable multiplex PCR assays is not straightforward.

Previously, we reported the development and successful application of a single tube QF-PCR 12-plex ${ }^{9}$ to 1336 prenatal samples, and a sex chromosome-targeted multiplex for assessing sex chromosome copy number. ${ }^{12}$ Here, we describe further development of the multiplex, including the replacement and addition of several microsatellite markers, resulting in a more informative and robust assay. Modification of sample processing procedures has improved service efficiency. We report the results from testing more than 7000 prenatal samples for trisomies 13, 18 and 21 and assess the extent to which this assay meets the demands of a rapid prenatal diagnostic service.

\section{Materials and methods}

The majority of prenatal samples (amniotic fluid, chorionic villi and fetal blood) were received from women at increased risk of a chromosome abnormality due to raised maternal age or following serum testing or the identification of anomalies by ultrasound scanning. A small number of samples were referred from women with a family history of a genetic disorder or excessive anxiety. Samples received by the Cytogenetics Laboratory from within the South East Thames region were tested using QF-PCR analysis of uncultured cells and karyotype analysis of G-banded chromosomes from cultured cells. Prenatal samples received from other London Cytogenetic Laboratories were tested using QF-PCR while the karyotype analysis was carried out by the referring laboratory. All prenatal samples were tested, regardless of sample quality.

Sample and DNA preparation protocols were as described previously, ${ }^{9}$ with the following modifications: two villi taken from different areas of the CVS were tested separately and the results were compared; blood-stained amniotic fluid samples were washed in water prior to DNA preparation; no other wash steps were carried out; between 100 and $300 \mu \mathrm{l}$ of InstaGene Matrix (Bio-Rad, Hercules, CA, USA) was used depending on cell pellet/villus size and the 
$56^{\circ} \mathrm{C}$ incubation step (InstaGene Matrix protocol) was not carried out.

The single-tube PCR assay was improved with the following modifications: some of the microsatellite markers and primer sequences were replaced (see Table 1 for full details) and PCR kits were prepared in advance, using a hot-start Taq buffer mix, tested and stored at $-20^{\circ} \mathrm{C}$. Additional markers were used if the multiplex proved to be uninformative for any chromosome (see Table 2 for details). PCR cycling conditions were: initial denaturation at $95^{\circ} \mathrm{C}$ for $15 \mathrm{~min}$, followed by 25 cycles of $94^{\circ} \mathrm{C}$ for $30 \mathrm{~s}$, $57^{\circ} \mathrm{C}$ for $1 \mathrm{~min} 30 \mathrm{~s}, 72^{\circ} \mathrm{C}$ for $1 \mathrm{~min} 30 \mathrm{~s}$, with final synthesis at $72^{\circ} \mathrm{C}$ for $20 \mathrm{~min}$. PCR products were analysed on a 3100 genetic analyser (Applied Biosystems, Foster City, CA, USA) and interpreted as described previously. ${ }^{9}$ To minimise the risk of sample mix-up, the protocol was designed to require just three tube-to-tube transfers; each transfer was carefully monitored. Confirmation of abnormal results for amniotic fluid samples was by an additional same-day QF-PCR test on media pour-off from one of the culture flasks (to confirm sample identification), rather than interphaseFISH; the testing of two villi negated the need for result confirmation for CVS.

\section{Results}

Since the rapid aneuploidy QF-PCR service began in June 2000, 7720 prenatal samples (6147 amniotic fluid samples, 1552 CVS and 21 fetal blood samples) have been tested for chromosomes 13, 18 and 21 copy number, followed by full karyotype analysis of cultured cells. The results from each test were compared, although for 22 CVS and 13 amniotic fluid samples no karyotype result was available, usually due to failure of the cell cultures. Of the samples tested, 7087 (91.8\%) were normal and 274 (3.5\%) had trisomy for

Table 1 Details of primers used in the QF-PCR multiplex

\begin{tabular}{|c|c|c|c|c|c|}
\hline Marker & Location & Size range $(b p)$ & Primer sequence $5^{\prime}-3^{\prime}$ & $\mu M$ & Reference/source \\
\hline $\begin{array}{l}\text { D13S305-F } \\
-R\end{array}$ & $13 q 13.3$ & $430-465$ & $\begin{array}{l}\text { HEX-GCCTGTTTGAGGACCTGTCGTTA } \\
\text { TGGTTATAGAGCAGTTAAGGCAC }\end{array}$ & 0.6 & GDB \\
\hline $\begin{array}{l}D 135628-F \\
-R\end{array}$ & $13 q 31.1$ & $425-470$ & $\begin{array}{l}\text { NED-TAACATTCATTGTCCCTTACAGAT } \\
\text { GCAAGGCTATCTAACGATAATTCA }\end{array}$ & 0.3 & GDB \\
\hline $\begin{array}{l}\text { D135634-F } \\
-R\end{array}$ & $13 q 21.33$ & $385-440$ & $\begin{array}{l}\text { 6-F-GGCAGATTCAATAGGATAAATAGA } \\
\text { GTAACСССTCAGGTTCTCAAGTCT }\end{array}$ & 0.6 & Pertl (1997) \\
\hline $\begin{array}{l}\text { D13S742-F } \\
-R\end{array}$ & $13 q 12.12$ & $235-315$ & $\begin{array}{l}\text { HEX-ATAACTGGGCTAGGAATGGAAATA } \\
\text { GACTTCCCAATTCAGGAGGACT }\end{array}$ & 0.6 & GDB \\
\hline $\begin{array}{l}\text { D18S978-F } \\
-R\end{array}$ & $18 q 12.3$ & $180-220$ & $\begin{array}{l}\text { NED-GTAGATCTTGGGACTTGTCAGA } \\
\text { GTCTCCCATGGTCACAATGCT }\end{array}$ & 0.12 & GDB \\
\hline $\begin{array}{l}\text { D18S386-F } \\
-R\end{array}$ & $18 q 22.1$ & $330-400$ & $\begin{array}{l}\text { HEX-TGAGTCAGGAGAATCACTTGGAAC } \\
\text { CTCTTCCATGAAGTAGCTAAGCAG }\end{array}$ & 0.6 & Pertl (1999) \\
\hline $\begin{array}{l}\text { D18S499-F } \\
-R\end{array}$ & $18 q 21.32$ & $390-410$ & $\begin{array}{l}\text { NED-AGATTACCCAGAAATGAGATCAG } \\
\text { GAAAATGTAGAAGTGAGTCACCT }\end{array}$ & 0.5 & GDB \\
\hline $\begin{array}{l}\text { D18S391-F } \\
-R\end{array}$ & $18 p 11.31$ & $140-180$ & $\begin{array}{l}\text { HEX-GGACTTACCACAGGCAATGTGACT } \\
\text { CTGGCTAATTGAGTTAGATTACAA }\end{array}$ & 0.2 & Gerken (1994) \\
\hline $\begin{array}{l}\text { D18S535-F } \\
-R\end{array}$ & $18 q 12.3$ & $455-500$ & $\begin{array}{l}\text { 6-F-CAGCAAACTTCATGTGACAAAAGC } \\
\text { CAATGGTAACСTACTATTTACGTC }\end{array}$ & 0.4 & Pertl (1997) \\
\hline $\begin{array}{l}\text { D21S11-F } \\
-R\end{array}$ & $21 q 21.1$ & $225-280$ & $\begin{array}{l}\text { 6-F-TITCTCAGTCTCCATAAATATGTG } \\
\text { GATGTTGTATTAGTCAATGTTCTC }\end{array}$ & 0.24 & Pertl (1996) \\
\hline $\begin{array}{l}\text { D21S1270-F } \\
-R\end{array}$ & $21 q 22.11$ & $285-340$ & $\begin{array}{l}\text { 6-F-CTATCCСACTGTATTATTCAGGGC } \\
\text { TGAGTCTCCAGGTTGCAGGTGACA }\end{array}$ & 0.8 & Bosch (1996) \\
\hline $\begin{array}{l}\text { D21S1411-F } \\
-R\end{array}$ & $21 q 22.3$ & $256-340$ & $\begin{array}{l}\text { ATAGGTAGATACATAAATATGATGA } \\
\text { NED-TATTAATGTGTGTCCTTCCAGGC }\end{array}$ & 0.8 & Pertl (1997) \\
\hline $\begin{array}{l}\text { D21S1435-F } \\
-R\end{array}$ & $21 q 21.3$ & $160-200$ & $\begin{array}{l}\text { 6-F-CCСТCTCAATTGTTTGTCTACC } \\
\text { ACAAAAGGAAAGCAAGAGATTTCA }\end{array}$ & 0.2 & GDB \\
\hline
\end{tabular}

Size ranges given are those used in Genotyper version 2.5. Some of the primer sequences have been redesigned from those published in order to create the multiplex detailed here. (GDB database: http://www.gdb.org) 
Table 2 Details of primers used in extra marker assays

\begin{tabular}{|c|c|c|c|c|c|}
\hline Marker & Location & Size range (bp) & Primer sequence $5^{\prime}-3^{\prime}$ & $\mu M$ & Reference/source \\
\hline $\begin{array}{l}\text { D13S252 F } \\
\text { R }\end{array}$ & $13 q 12.1$ & $270-320$ & $\begin{array}{l}\text { 6-F-GCA GAT GTA CTG TTT TCC TAC CAA } \\
\text { AGA TGG TAT ATT GTG GGA CCT TGT }\end{array}$ & 0.4 & GDB \\
\hline $\begin{array}{l}\text { D13S762 F } \\
\text { R }\end{array}$ & $13 q 31-q 32$ & $270-320$ & $\begin{array}{l}\text { HEX-AAT GAG ATT GCT GGG TCA GA } \\
\text { GTG GCT CCA TGG AAA TTT A }\end{array}$ & 0.4 & GDB \\
\hline $\begin{array}{l}\text { D18S1002 F } \\
\text { R }\end{array}$ & $18 q 11-q 11$ & $340-370$ & $\begin{array}{l}\text { 6-F-GTT TGA TGG GAG GAA GCT ATC TAT } \\
\text { GTG AAG TAG CGG AAG GCT GTA AT }\end{array}$ & 0.4 & GDB \\
\hline $\begin{array}{l}\text { IFNAR F } \\
\text { R }\end{array}$ & $21 q 22.1$ & $370-410$ & $\begin{array}{l}\text { NED- CATTTGATCTTAGCCATCTATTGC } \\
\text { ACTATGCAGCCATTTGAAAGACTA }\end{array}$ & 0.4 & Mclnnis (1991) \\
\hline $\begin{array}{l}\text { D21S226 F } \\
\text { R }\end{array}$ & $21 q 22.1$ & $440-470$ & $\begin{array}{l}\text { 6-F-GCAAATTTGTGGATGGGATTAACAG } \\
\text { AAGCTAAATGTCTGTAGTTATTCT }\end{array}$ & 0.4 & Chumakov (1992) \\
\hline
\end{tabular}

The markers are amplified in three chromosome-specific assays. Size ranges given are those used in Genotyper version 2.5. Some of the primer sequences have been redesigned from those published in order to create the multiplex detailed here. (GDB database: http://www.gdb.org)

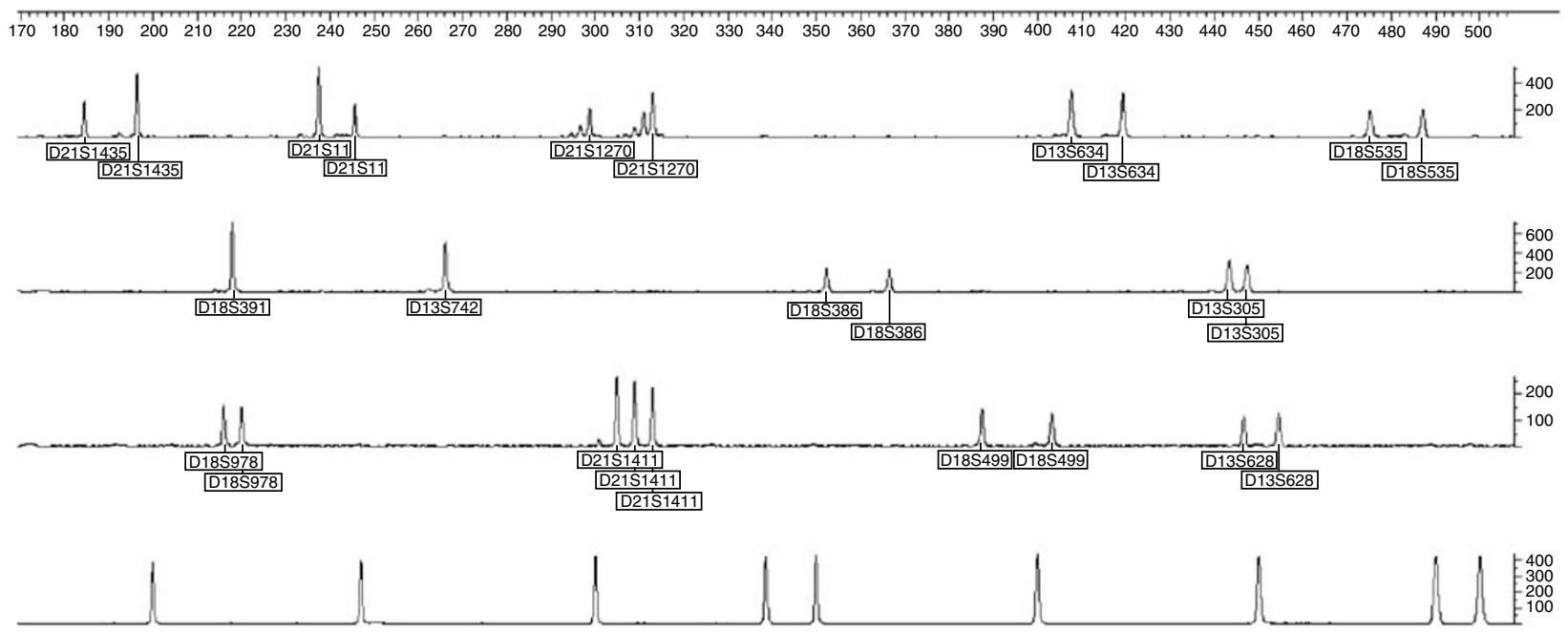

Figure 1 Genotyper profile of a trisomy 21 sample. Fragment size in bp shown on the horizontal axis, arbitrary fluorescence units shown on the vertical axis. Peaks are labelled with marker name. Chromosome 21 markers exhibit two alleles in a $1: 2$ ratio (D21S1435 and D21S1270) or a 2:1 ratio (D21S11), or three alleles (D21S1411); chromosomes 13 and 18 markers exhibit normal 1:1 ratios or uninformative (homozygous) results (D18S391 and D13S742).

chromosome 21 (Figure 1), 105 (1.4\%) trisomy for chromosome $18,45(0.6 \%)$ trisomy for chromosome 13 ; the remaining $13(0.2 \%)$ were triploid, one of which also had tetrasomy for chromosome 21 . Two samples were found to have partial chromosome imbalance as shown by both normal and trisomy results for markers on the same chromosome; the karyotypes of these two samples were subsequently found to be $46, \mathrm{XY}, \operatorname{der}(4) \mathrm{t}(4 ; 18)(\mathrm{q} 35 ; \mathrm{q} 21.3)$ and 46,XY,der(19)t(19;21)(p13.3;q21). There were no discrepancies between the QF-PCR and karyotype results for all normal and nonmosaic trisomy samples.

For 167 (2.16\%) samples, no QF-PCR result was available for at least one of the three chromosomes. Of these, 30 samples $(0.39 \%$ of total samples tested) were uninformative for all markers on a single chromosome, although most of these were tested with the original, less informative, multiplex assay. With the modified multiplex and extra marker assays $0.05 \%$ of samples were uninformative for a single chromosome. DNA from seven samples $(0.09 \%$ of total samples tested) failed to amplify consistently, and therefore no rapid result was available. All of these were brown amniotic fluid samples. The majority of referrals (130; $1.68 \%$ of total samples tested) that did not receive a QF-PCR result were blood-stained amniotic fluid samples (although most blood-stained samples did receive a QFPCR result). The amount of blood in these 130 samples ranged from a low level that was only visible in the cell pellet, to high-level staining of the fluid. The presence of two genotypes, both at high levels, prevented result interpretation due to allele ratios outside of the normal 
range. The pattern was characteristic of a contaminating maternal genotype with the shared fetal-maternal allele being approximately equal to the sum of the fetal-specific and maternal-specific alleles, and an absence of four-allele results (Figure 2). These samples were reported as unsuitable for QF-PCR analysis, with a karyotype analysis result to follow. Follow-up genotype analysis of cultured cells from these samples showed the maternal genotype to be lost in nearly all cases (unpublished results). A small number of amniotic fluid samples were heavily bloodstained (red in colour). In those that were found to have a single female genotype, a maternal blood sample was requested and tested to determine the cell origin. However, the majority of blood-stained amniotic fluid samples that showed MCC contained just a small amount of blood, visible in the cell pellet, and exhibited a low-level second genotype. If informative marker results exhibited consistent results (normal allele ratios of $0.8-1.4$ or trisomic allele patterns with three equal peaks or allele ratios less than 0.65 or greater than 1.8), results were issued on these samples.

In all, 12 samples were found to have a submicroscopic imbalance, evident as a single marker result consistent with trisomy while all other informative markers were normal (Figure 3), as was the karyotype. Of these, seven were duplicated for at least the sequence represented by the D13S742 marker (13q12.12), three were duplicated for at least the sequence represented by the D13S634 marker (13q21.33) and two were duplicated for at the least sequence represented by the D18S386 marker (18q22.1). QF-PCR analysis of parental blood samples showed all of these to be inherited and therefore unlikely to be of clinical significance. Further work is underway to characterise the location and size of these imbalances.

Overall, 15 samples were found to be mosaic for trisomy or triploidy by QF-PCR and/or karyotype analysis. Separate analysis of two villi increased the chances of detecting mosaicism. Mosaicism detected by QF-PCR was evident as skewed allele ratios and/or minor third alleles for all markers for a particular chromosome (mosaic trisomy) (Figure 4), or all chromosomes (mosaic triploidy). Meiosisderived abnormal cell lines (exhibiting three alleles) present at $15 \%$ of the cell population were detected by QF-PCR. QF-PCR of uncultured cells identified the abnormal cell line in 12 of the 15 samples, while karyotype analysis also identified the abnormal cell line in 12 samples. Differences between QF-PCR and karyotype results in mosaic cases are likely to be due to the testing of different populations of cell types.

Somatic microsatellite mutations $(\mathrm{SMMs})^{13}$ were observed in 69 CVS and seven amniotic fluid samples, usually evident as a three-allele pattern with the two peak areas representing the mutated allele approximately equal to the stable allele. Conclusions were based on the other informative markers and the SMMs were not reported.

The results of the QF-PCR assay were faxed to the referring centre as soon as they were available. In 2003, $97.0 \%$ of results were reported on the working day following receipt of the sample, $2.7 \%$ were reported 2 working days following receipt of the sample and $0.3 \%$ were reported on 3 or more working days following sample receipt. The average reporting time for karyotype analysis of prenatal samples in our laboratory in 2003 was 15 days.

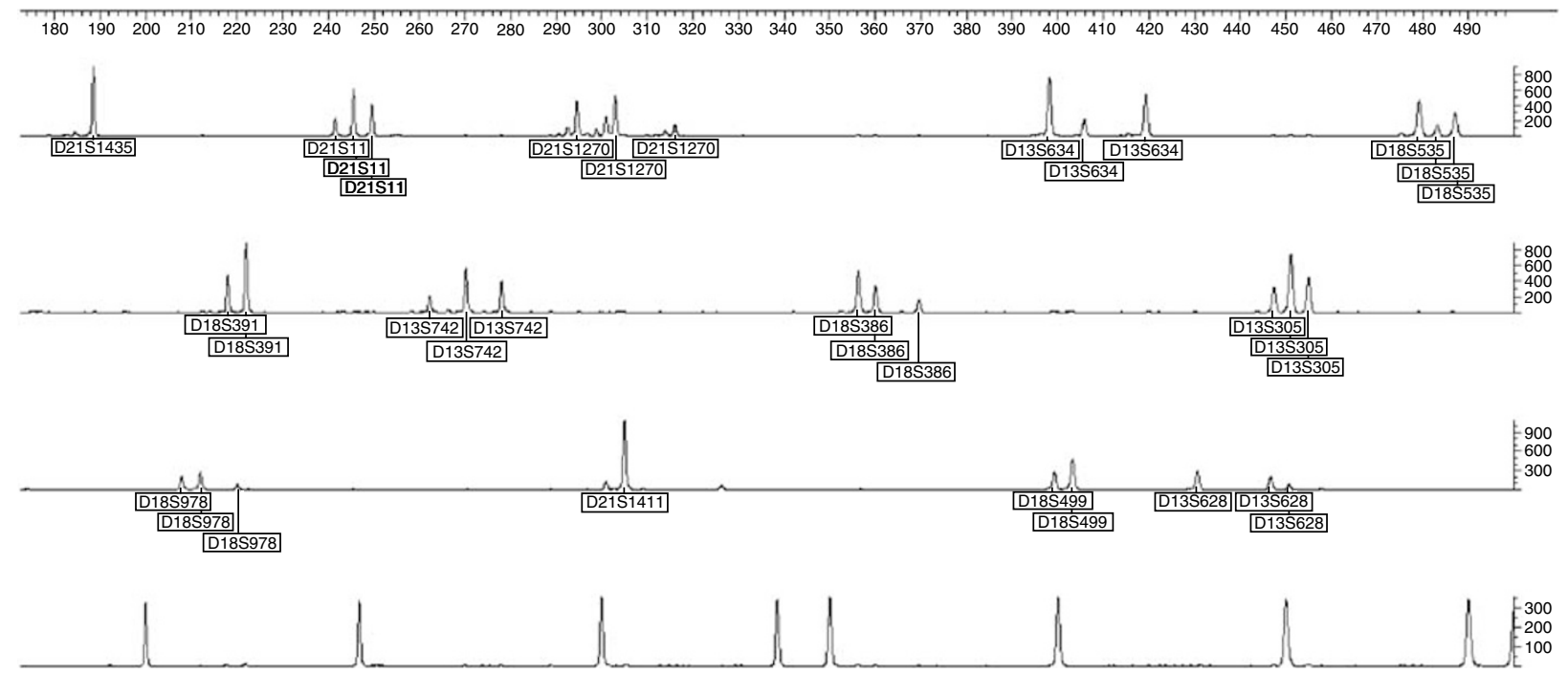

Figure 2 Genotyper profile of a blood-stained amniotic fluid sample with high-level MCC. The profile is labelled as described in Figure 1. Characteristic triallelic results are seen for most markers and show the combined fetal-maternal allele, and smaller fetal- and maternal-specific alleles. Diallelic results with skewed allele ratios are present (D18S391 and D18S499) and homozygous results (D21S1435 and D21S1411). There are no four-allele results. 


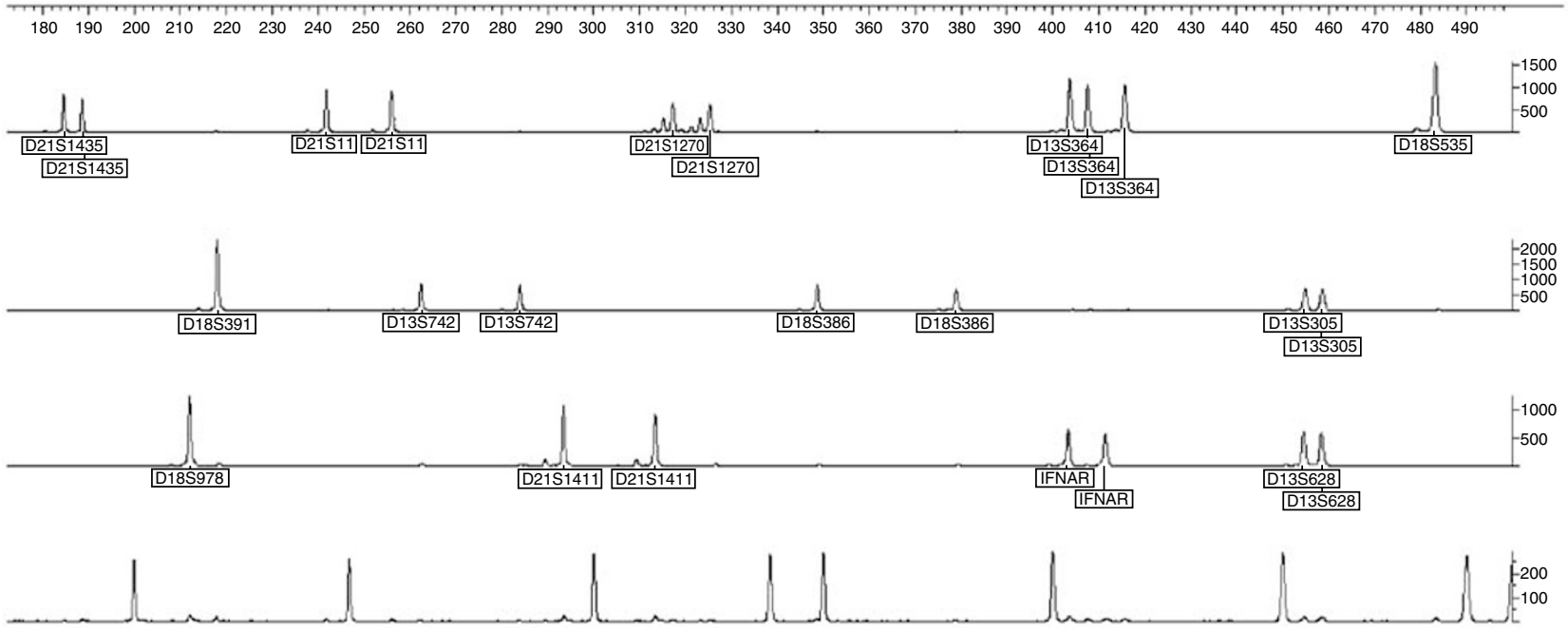

Figure 3 Genotyper profile of sample with duplication of a single locus. The profile is labelled as described in Figure 1. The D13S634 triallelic result represents the duplication, while all other chromosome 13 marker results exhibit normal 1:1 ratios. The duplication was subsequently found to be submicroscopic and inherited from the mother.
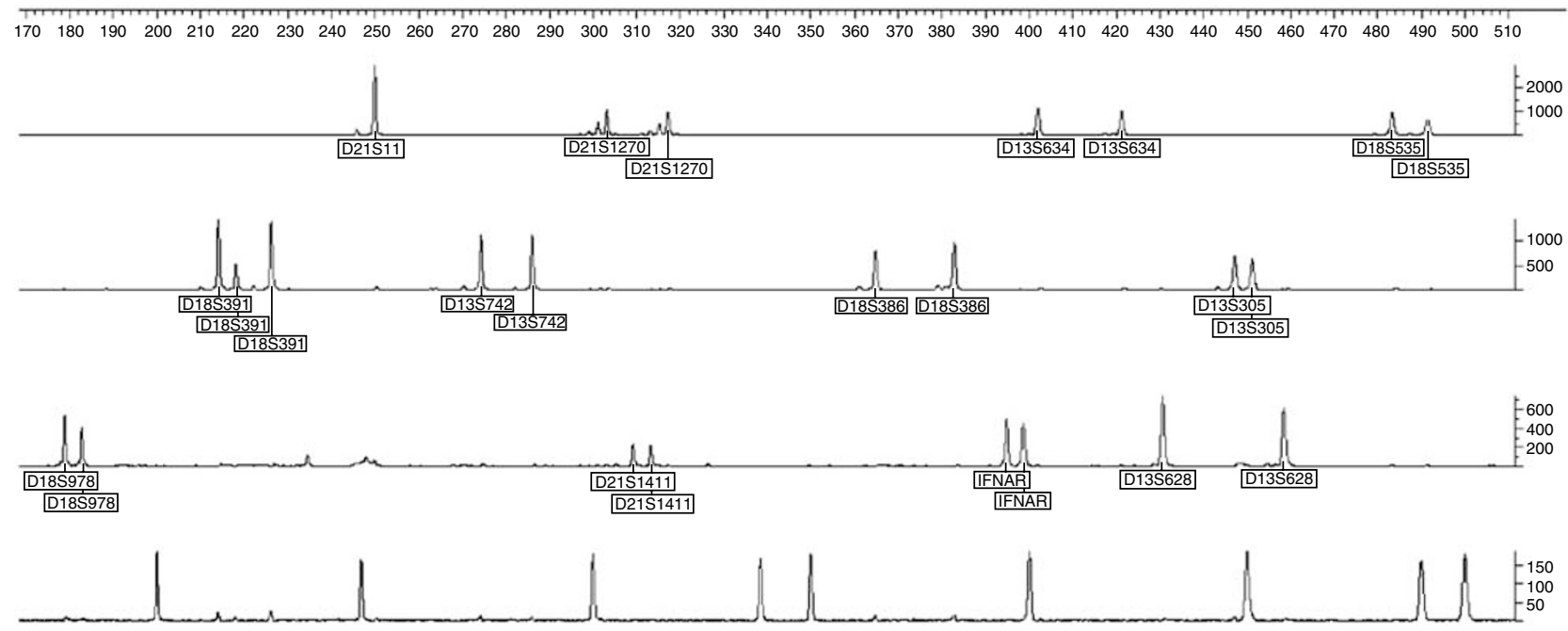

Figure 4 Genotyper profile of a chorionic villus sample with trisomy 18 mosaicism. The profile is labelled as described in Figure 1. Three chromosome 18 markers show diallelic results with skewed ratios (D18S535, D18S386 and D18S978). The D18S391 marker exhibits three alleles, with the lowest allele representing the trisomy cell line, and shows the nondisjunction event to be meiotic in origin. The abnormal cell line accounts for approximately $40 \%$ of the cell population in two villi tested. Karyotype analysis of cultured cells found all cells to be trisomy 18.

\section{Discussion}

We describe an improved and updated aneuploidy diagnostic assay using a one-tube QF-PCR test and the prospective testing of more than 7000 prenatal samples, the largest QF-PCR data set currently reported. New primer sequences and modified protocols have improved service efficiency. There were no false-positive or false-negative results for nonmosaic trisomy. The amplification failure rate was exceptionally low at $0.09 \%$ of samples and the markers in the modified multiplex assay were informative for $99.95 \%$ of samples.

Microsatellite genotype analysis has the advantage of identifying the presence of a second cell line. This was demonstrated by the efficient detection of both mosaicism and MCC; cell lines contributing at least $20 \%$ of the total cell population were confidently identified. The abnormal cell line was detected by QF-PCR in 12 of 15 trisomy mosaic samples and by karyotype analysis in 12 of the 15 . 
Differences between the results are likely to be due to the different cell populations analysed. Samples (1.68\%), most of which were blood-stained amniotic fluid, were reported as unsuitable due to MCC. These exhibited either two genotypes, both at significant levels that prevented confident result interpretation, or a single female genotype, identified as maternal following analysis of a maternal blood sample. Identification of MCC reduces the risk of a misdiagnosis based on analysis of maternal cells.

Ambiguous results are likely to increase parental anxiety. Here, samples that required follow-up analysis of parental samples for result interpretation were rare, limited to those where the origin of the tested cells was in doubt due to poor sample quality or samples found to have imbalance at a single locus. All 12 submicroscopic duplications were shown to be inherited and therefore unlikely to be of clinical significance. Other ambiguous results such as SMMs and primer site polymorphisms were resolved by additional PCR analysis of the sample and conclusions based on results from other markers.

The service has proven to be both rapid and reliable with $97 \%$ of samples reported the working day following sample receipt. To facilitate this, sample processing was simplified and batches of single-tube assays were prepared, tested and stored in advance. The service is suited to high-throughput as more than 20 prenatal samples can be prepared and setup for PCR in $1 \mathrm{~h}$ and analysis is semi-automated. A 3100 genetic analyser is able to process 16 samples/h and these can be analysed in less than an hour by a single scientist. Consumables costs are approximately $£ 5 /$ sample; however, the initial capital cost of a genetic analyser is considerable, and development and validation of the multiplex can be technically demanding with no commercial kits currently available.

QF-PCR has been implemented at a number of other diagnostic laboratories as a validated service; ${ }^{7,8,10,11}$ these laboratories use a range of microsatellite markers multiplexed together in different ways. Although the design of individual assays in these laboratories may affect service efficiency, the assays are reported as robust and reliable, indicating that QF-PCR is a technique suitable for application at different sites.

QF-PCR compares favourably with interphase-FISH, particularly when cost and labour resources are evaluated. ${ }^{14}$ However, interphase-FISH has proven to be an accurate and rapid method with a predicted false-positive rate of less than 1 in 30000 cases and a false-negative rate of less than 1 in $4000,{ }^{4}$ although some centres have reported higher rates of misdiagnosis. ${ }^{15-17}$ Most misdiagnoses are due to MCC or cross-hybridization of probes to other loci. Single FISH probes may also fail to detect regional imbalance elsewhere on the chromosome and have been reported to give false-negative results. ${ }^{15,18}$ Conversely, a reported false-positive trisomy 18 result was due to an insertion of pericentromeric alpha satellite DNA into the proximal long arm of chromosome $9 .{ }^{19}$ Failure to detect regional imbalance also applies to some molecularbased approaches, although in the case of QF-PCR several sequences (either spread along the chromosome or targeted to the Down's critical region) are investigated, minimising the risk of misdiagnosis due to a small regional imbalance. Using interphase-FISH it is not possible to detect the presence of maternal cells in samples from female fetuses. Mosaicism is detectable, although detection levels will depend on the number of analysed cells. Larger samples are required than for QF-PCR; overall the reported failure rates (hybridisation failure or inadequate volume of amniotic fluid) vary from $0.71^{4}$ to $3 \% .{ }^{17}$

Preliminary data for the application of two other PCRbased approaches to rapid prenatal aneuploidy diagnosis have been published. The first was described in $1997,{ }^{20}$ as homologous gene quantitative PCR (HGQ-PCR). A single pair of primers was used to simultaneously amplify sequences from homologous genes on chromosomes 1 and 21. The relative amount of each sequence, a $345 \mathrm{bp}$ sequence from chromosome 1 and a $185 \mathrm{bp}$ sequence from chromosome 21, was used to determine chromosome copy number. In total, 34 samples with trisomy 21 and 100 normal controls were tested and the difference between the average dosage ratio of the two groups was found to be highly significant. DNA from blood samples and cultured amniocytes was tested. A modification of this approach has been described, ${ }^{21}$ where analysis of the relative copy number of paralogous genes used pyrosequencing technology. The development of assays for chromosomes 13, 18, $21, \mathrm{X}$ and $\mathrm{Y}$ are reported, but details are not yet published.

The second quantitative PCR approach uses different primer pairs to multiplex three nonparalogous sequences (one each from chromosomes 13, 18 and 21). ${ }^{22}$ Comparison of the relative amplification of these sequences was used to determine genomic copy number; 400 uncultured amniotic fluid samples were tested and all results were in agreement with the results of the karyotype analysis. A more automated approach is the use of real-time PCR to assess the relative amounts of amplified nonparalogous sequences from different chromosomes. ${ }^{23}$ In a small scale pilot study, sequences from chromosomes 12 and 21 were compared and DNA from cultured amniocytes and peripheral blood was tested. Nine of 11 normal samples and nine of 10 trisomy 21 samples were correctly identified. Although these studies were all preliminary, they have the potential advantage of being informative for all samples, as microsatellite markers are not required. Furthermore, the real-time PCR strategy does not require a separate analysis step, and therefore reduces the risk of contamination and sample mix-up. However, these quantitative PCR approaches have some limitations: while the QF-PCR approach described in this paper exploits a 2:1 diallelic ratio and/or a qualitative triallelic result, the two other PCR approaches require a more subtle 3:2 dosage 
ratio to identify trisomy and for this reason may be less sensitive for the detection of trisomy mosaicism; triploidy and MCC are not detectable if the fetus is female (and in these studies sex chromosome copy number was not investigated); comparison of sequences amplified with different primer sets in the nonparalogous approach is likely to make it more susceptible to variation in sample quality, although one reported failure rate ${ }^{22}$ was just $1 \%$ and finally, additional sequences should be included in these assays to remove the risk of misdiagnosis based on primer site polymorphisms or small segment imbalance.

The investigation of tens to hundreds of loci simultaneously would be a useful tool for the analysis of postnatal samples. However, for prenatal diagnosis the testing of ever more loci increases the likelihood of ambiguous results such as locus-specific or regional duplications and deletions with unknown clinical significance. There is a convincing argument that for prenatal diagnosis, targeted testing of well-defined sequences and loci has benefits over a whole genome screen. ${ }^{24}$ Nevertheless, two approaches are being developed and evaluated for the diagnosis of genomic copy number change at numerous loci in prenatal samples. The first is multiplex ligation-dependent probe amplification (MLPA), a novel technique that is being validated in many centres for the diagnosis of genomic copy number changes. ${ }^{25}$ The principle of this approach is the hybridisation of two oligos to adjacent genomic sequences followed by their ligation to form a single sequence. Oligos complementary to many different sequences can be hybridised and ligated in a single assay. Each oligo includes a $5^{\prime}$ forward or reverse common primer sequence for amplification of the ligated sequences; these sequences are of different lengths to allow size separation of the products on a genetic analyser. The amount of each amplified sequence relative to the other sequences can then be used to predict sequence copy number. Although the potential of MLPA as a diagnostic test is still being assessed, for many applications it has the advantage of being able to investigate the genomic copy number of up to 50 loci in a single assay and commercial MLPA kits are available, although sold for research use only (MRCHolland). One such kit has been designed for the identification of chromosomes 13, 18, 21, X and $\mathrm{Y}$ aneuploidy and contains eight probes specific for each of the chromosomes 13, 18 and 21. This assay has recently been assessed in a blind, prospective study that tested almost 500 amniotic fluid samples. ${ }^{26}$ The results for nonblood-stained samples were promising, with no falsepositive or false-negative results for trisomy 13,18 or 21 . Results were available within $48 \mathrm{~h}$, which included a $16 \mathrm{~h}$ hybridisation step. In all, $8 \%$ of samples required repeat analysis following concentration of DNA by ethanol precipitation. Some single probe results were found to be inconsistent with other chromosome-specific probes: individual loci indicated normal copy number in aneuploid samples or abnormal copy number in normal samples. As analysis of individual loci gave discrepant results, the median value for each chromosome probe set (using all eight probes) was used to determine chromosome copy number. This analysis removes some benefit of using multiple probes and is likely to fail to detect regional genomic imbalance such as unbalanced translocation products. Furthermore, the normal and abnormal threshold values calculated with $99 \%$ confidence limits for the median chromosome probe set value appear to overlap. Finally, triploidy and MCC are not identified if the fetus is female, and sensitivity to intermediate copy number changes representing mosaicism is likely to be low given the threshold limits. The authors ${ }^{26}$ recognise limitations in this MLPA approach by describing the assay as a screening test rather than a diagnostic test.

Comparative genomic hybridisation using arrays has the potential to be more informative, investigating copy number of hundreds of loci. Test DNA and control DNA are differentially labelled, mixed and hybridised to arrays of target clones. The ratio of the test to control DNA represents genomic copy number at that locus. The potential of arrays is currently being realised in the postnatal field, ${ }^{27}$ and preliminary studies have been carried out using DNA from prenatal samples. ${ }^{28,29}$ Microarray CGH would fail to identify MCC and triploidy in female prenatal samples, and the lengthy hybridisation step and high cost of commercial arrays may restrict their use for rapid trisomy diagnosis, especially if ambiguous and/or inconclusive results give rise to extensive repeat testing and associated patient anxiety.

The possibility of using QF-PCR as a stand-alone test to replace full karyotype analysis in pregnancies without ultrasound abnormalities has been suggested. ${ }^{24,30}$ However, careful consideration by all involved professional groups will be necessary before such a step is likely to be taken. The current rationale for rapid prenatal aneuploidy testing is to improve pregnancy management, or provide rapid reassurance in cases with a normal result and no ultrasound abnormalities. Any potential strategy must therefore be thoroughly validated and proven to be accurate and robust in spite of the specific difficulties associated with testing prenatal samples. Furthermore, the assay should be able to detect mosaicism and MCC in order to minimise the risk of a misdiagnosis. Finally, the service must provide consistently rapid results and should be costeffective if it is to be a feasible adjunct to karyotype analysis. The data presented in this paper demonstrate that QF-PCR is the current method of choice for rapid prenatal aneuploidy diagnosis.

\section{Acknowledgements}

We acknowledge the technical assistance of Alysia Hallam, and the contribution of the Cytogenetics Department Prenatal Team. 


\section{References}

1 NEQAS: National External Quality Assessment Scheme in Clinical Cytogenetics; Annual Report: 2000.

2 Smith K, Lowther G, Maher E, Hourihan T, Wilkinson T, Wolstenholme J: The predictive value of findings of the common aneuploidies, trisomies 13, 18 and 21, and numerical sex chromosome abnormalities at CVS: experience from the ACC U.K. Collaborative Study. Association of Clinical Cytogeneticists Prenatal Diagnosis Working Party. Prenat Diagn 1999; 19: $817-826$.

3 Klinger K, Landes G, Shook D et al: Rapid detection of chromosome aneuploidies in uncultured amniocytes by using fluorescence in situ hybridization (FISH). Am J Hum Genet 1992; 51: $55-65$

4 Tepperberg J, Pettenati MJ, Rao PN et al: Prenatal diagnosis using interphase fluorescence in situ hybridization (FISH): 2-year multi-center retrospective study and review of the literature. Prenat Diagn 2001; 21: 293-301.

5 Mansfield ES: Diagnosis of Down syndrome and other aneuploidies using quantitative polymerase chain reaction and small tandem repeat polymorphisms. Hum Mol Genet 1993; 2: $43-50$.

6 Pertl B, Yau SC, Sherlock J, Davies AF, Mathew CG, Adinolfi M: Rapid molecular method for prenatal detection of Down's syndrome. Lancet 1994; 343: 1197-1198.

7 Levett LJ, Liddle S, Meredith R: A large-scale evaluation of amnioPCR for the rapid prenatal diagnosis of fetal trisomy. Ultrasound Obstet Gynecol 2001; 17: 115-118.

8 Bili C, Divane A, Apessos A et al: Prenatal diagnosis of common aneuploidies using quantitative fluorescent PCR. Prenat Diagn 2002; 22: 360-365.

9 Mann K, Fox SP, Abbs SJ et al: Development and implementation of a new rapid aneuploidy diagnostic service within the UK National Health Service and implications for the future of prenatal diagnosis. Lancet 2001; 358: 1057-1061.

10 Cirigliano V, Lewin P, Szpiro-Tapies S, Fuster C, Adinolfi M: Assessment of new markers for the rapid detection of aneuploidies by quantitative fluorescent PCR (QF-PCR). Ann Hum Genet 2001; 65: 421-427.

11 Schmidt W, Jenderny J, Hecher K et al: Detection of aneuploidy in chromosomes X, Y, 13, 18 and 21 by QF-PCR in 662 selected pregnancies at risk. Mol Hum Reprod 2000; 6: 855-860.

12 Donaghue C, Roberts A, Mann K, Ogilvie CM: Development and targeted application of a rapid QF-PCR test for sex chromosome imbalance. Prenat Diagn 2003; 23: 201-210.

13 Mann K, Donaghue C, Ogilvie CM: In vivo somatic microsatellite mutations identified in non-malignant human tissue. Hum Genet 2003; 114: 110-114.

14 Hulten MA, Dhanjal S, Pertl B: Rapid and simple prenatal diagnosis of common chromosome disorders: advantages and disadvantages of the molecular methods FISH and QF-PCR. Reproduction 2003; 126: 279-297.
15 Liehr T, Beensen V, Hauschild R et al: Pitfalls of rapid prenatal diagnosis using the interphase nucleus. Prenat Diagn 2001; 21: 419-421.

16 Liehr T, Schreyer I, Neumann A et al: Two more possible pitfalls of rapid prenatal diagnostics using interphase nuclei. Prenat Diagn 2002; 22: 497-499.

17 Weremowicz S, Sandstrom DJ, Morton CC, Niedzwiecki CA, Sandstrom MM, Bieber FR: Fluorescence in situ hybridization (FISH) for rapid detection of aneuploidy: experience in 911 prenatal cases. Prenat Diagn 2001; 21: 262-269.

$18 \mathrm{Yu} \mathrm{C}$, Bofill B: False negative interphase FISH assay on three prenatal cases of trisomy 18. Am J Hum Genet 2003; 73: 601.

19 Singh-Kahlon PD, Gadi I, Tepperberg J et al: Conflicting results between prenatal interphase FISH \& G-banded chromosome diagnoses: implications for clinical decisions. Am J Hum Genet 2003; 73: 312 .

20 Lee HH, Chang JG, Lin SP, Chao HT, Yang ML, Ng HT: Rapid detection of trisomy 21 by homologous gene quantitative PCR (HGQ-PCR). Hum Genet 1997; 99: 364-367.

21 Deutsch S, Choudhury U, Sylvan A, Antonarakis SE: Detection of trisomy 21 and other aneuploidies by Paralogous gene quantification. Am J Hum Genet 2003; 73: 318.

22 Rahil H, Solassol J, Philippe C, Lefort G, Jonveaux P: Rapid detection of common autosomal aneuploidies by quantitative fluorescent PCR on uncultured amniocytes. Eur J Hum Genet 2002; 10: 462-466.

23 Zimmermann B, Holzgreve W, Wenzel F, Hahn S: Novel real-time quantitative PCR test for trisomy 21. Clin Chem 2002; 48: 362-363.

24 Ogilvie CM: Prenatal diagnosis for chromosome abnormalities: past, present and future. Pathol Biol (Paris) 2003; 51: 156-160.

25 Schouten JP, McElgunn CJ, Waaijer R, Zwijnenburg D, Diepvens F, Pals G: Relative quantification of 40 nucleic acid sequences by multiplex ligation-dependent probe amplification. Nucleic Acids Res 2002; 30: e57.

26 Slater HR, Bruno DL, Ren H, Pertile M, Schouten JP, Choo KH: Rapid, high throughput prenatal detection of aneuploidy using a novel quantitative method (MLPA). J Med Genet 2003; 40: 907-912.

27 Carter NP, Fiegler H, Piper J: Comparative analysis of comparative genomic hybridization microarray technologies: report of a workshop sponsored by the Wellcome Trust. Cytometry 2002; 49: $43-48$.

28 Rauen KA, Norton ME, Segraves R et al: Array comparative genomic hybridization: applications in genetic medicine. Am J Hum Genet 2003; 73: 204

29 Pestova E, Ruffalo T, Marble T, Kucas M, Wilber K, King W: Reproducibility and performance of Vysis GSA300 platform for pre- and post-natal array-based CGH diagnostics. Am J Hum Genet 2003; 73: 310

30 Leung WC, Lau ET, Lao TT, Tang MH: Can amnio-polymerase chain reaction alone replace conventional cytogenetic study for women with positive biochemical screening for fetal Down syndrome? Obstet Gynecol 2003; 101: 856-861. 